\title{
Fabrication of biomolecule-copolymer hybrid nanovesicles as energy conversion systems
}

\author{
Dean Ho ${ }^{1,2,3,4}$, Benjamin Chu ${ }^{1,4}$, Hyeseung Lee ${ }^{1}$, Evan K Brooks ${ }^{1}$, \\ Karen Kuo $^{1}$ and Carlo D Montemagno ${ }^{1,3}$ \\ ${ }^{1}$ Department of Bioengineering, University of California at Los Angeles, 7523 Boelter Hall, \\ 420 Westwood Plaza, Los Angeles, CA 90095, USA \\ 2 Department of Electrical Engineering, California Institute of Technology, 1200 E. \\ California Boulevard, Mail Code 136-93, Pasadena, CA 91125, USA \\ E-mail: deanh@caltech.edu and cdm@seas.ucla.edu
}

Received 10 May 2005, in final form 8 September 2005

Published 11 November 2005

Online at stacks.iop.org/Nano/16/3120

\begin{abstract}
This work demonstrates the integration of the energy-transducing proteins bacteriorhodopsin (BR) from Halobacterium halobium and cytochrome $c$ oxidase (COX) from Rhodobacter sphaeroides into block copolymeric vesicles towards the demonstration of coupled protein functionality. An ABA triblock copolymer-based biomimetic membrane possessing UV-curable acrylate endgroups was synthesized to serve as a robust matrix for protein reconstitution. BR-functionalized polymers were shown to generate light-driven transmembrane $\mathrm{pH}$ gradients while $\mathrm{pH}$ gradient-induced electron release was observed from COX-functionalized polymers. Cooperative behaviour observed from composite membrane functionalized by both proteins revealed the generation of microamp-range currents with no applied voltage. As such, it has been shown that the fruition of technologies based upon bio-functionalizing abiotic materials may contribute to the realization of high power density devices inspired by nature.
\end{abstract}

(Some figures in this article are in colour only in the electronic version)

\section{Introduction}

Foundational studies of energy-transducing proteins have provided important information regarding their basic functionality [1-4]. Based on these fundamental works, the fabrication of protein-based devices can be explored [5, 6]. Enzymes including bacteriorhodopsin (BR) [7], cytochrome $c$ oxidase (COX) [8], photosystem I (PSI) [9], as well as adenosine triphosphate (ATP) synthase [10] function as energy transducers. Here we show that the directed integration of BR and COX into a robust, polymeric membrane technology and the addition of an electron transfer mediator in solution offer the opportunity of producing coordinated behaviour based on coupled protein functionality. More specifically, we have shown that

\footnotetext{
3 Authors to whom any correspondence should be addressed.

4 These authors contributed equally to this work.
}

BR functions as a light-activated ion pump to develop proton gradients that partially reverse COX, inducing the oxidation of its iron/copper centre to the cytochrome $c$ (cyt $c$ ) acceptor $[5,6,11,12]$. Using composite (protein-functionalized) polymeric vesicles of 250-300 nm diameter [13-15], we have demonstrated the use of BR as a light-dependent switch to induce electron release by COX for microamp-range current production with no applied voltage. Cycles of light/dark conditions resulted in increases/decreases, respectively, of current generation. These findings have demonstrated the feasibility of using this coupled protein activity as a biologically active anodic source of electrons for applications in biosolar cell technology.

Functionalization of biopolymers with energy transduction proteins utilizes the emerging concept of synthetic biology towards the production of practical devices based on inherent 
molecular function. The utilization of these membrane proteins harnesses key advantages that nature has evolved into producing perhaps some of the most efficient energetic systems on the planet. For example, artificially modified photosynthetic reaction centres have reached internal efficiencies as high as $80 \%$ with a quantum yield of converting sunlight energy into the energy of separated charges at a level near 1 [16]. In addition, the retinal chromophore, which serves as the lightdependent element of BR, possesses a quantum yield of 0.7, and a high molar extinction coefficient, as well as a short time interval required for isomerization that resides within the picosecond range [16]. These characteristics make a compelling case for retinal as an ideal energy converter as well.

The development of biological energy sources has generated significant interest in the energetics community. Das et al have previously demonstrated an interesting biosolar study of measuring light-dependent current production as a function of applied voltage using the plant protein photosystem I (PSI) while interfaced with microfabricated devices [17]. This work utilized a single light-driven protein energy transducer that was integrated with solid-state devices to produce an estimated performance efficiency of $12 \%$ and current densities of $0.12 \mathrm{~mA} \mathrm{~cm}^{-2}$ [17]. Furthermore, Kim et al demonstrated the use of glucose oxidase to transduce glucose fuel into electricity towards applications in in vivo implantation of biofuel cells for medical implants given the abundance of glucose within the human body [18]. Utilizing a two-electron electrooxidation process in glucose oxidase, and by 'wiring' these proteins to electrodes using conductive hydrogels, current and power outputs as high as $8.3 \mu \mathrm{A}$ and $4.3 \mu \mathrm{W}$, respectively, were observed while operating at physiological conditions of $37^{\circ} \mathrm{C}$. The work detailed here utilizes a protein-coupling process and the light-activated characteristics of BR to drive COX partial reversal to produce a current output, current density, and power as high as $10 \mu \mathrm{A}$, $0.14 \mathrm{~mA} \mathrm{~cm}^{-2}$, and $2.4 \mu \mathrm{W}$, respectively, with an estimated efficiency of $6 \%$. Furthermore, the modality of generating protein-functionalized nanovesicles supported by a solutionbased electron transfer mediator served as a rapid fabrication process for the biosolar system. While the roadmap for biomolecule-based energy source development is still in its nascent stage, these studies, along with the work presented in this paper, demonstrate the potential for using biology as a source of harnessed energy by engineering robust materials and devices that produce electrical energy outputs.

The work reported here has measured current output through combined $\mathrm{BR}$ and $\mathrm{COX}$ activity in a polymeric membrane. The successful engineering of this system has been characterized by a multitude of fabrication strategies and modalities beginning with the development of a biomimetic triblock copolymer with enhanced robustness over conventional lipid membranes via UV-crosslinked acrylatebased endgroups. In addition, we developed nanoscale vesicle reactors as the systemic architecture to enable rapid integration of $\mathrm{BR}$ and $\mathrm{COX}$ through a spontaneous vesicle formation process. Furthermore, given that the protein-coupling process is gradient dependent, a vesicular configuration allowed for a more robust system not subject to potential system disruption due to defects commonly observed in planar systems. Following polymeric characterization, the functional protein components were integrated according to the BRinduced unidirectional transport of protons to the vesicle exterior, and accomplished through a $\mathrm{pH}$ gradient-dependent vectorial insertion modality [19]. Vectorial catalysis by BR was then utilized to drive COX in a partially reversed reaction, altering its normal proton pumping mechanism, to become a source of electron release. To enable electron transport to the electrodes, a cytochrome $c$ mediator was used due to rapid electron transfer rates between heme groups (COX) and mediators over distances as high as $14 \AA$ [20].

The fully assembled system has successfully demonstrated light-dependent current generation through protein coupling with no applied voltage, satisfying the anodic component requirements for potential integration into a bioenergy source. It has been shown that polymer membrane fluidity, the ability to transport water, and hence, biomimicry, are dependent upon membrane thickness and molecular weight. As such, the advantages of utilizing membrane-bound energy transducers as the core functional component of the system were further enhanced by integrating them into endgroup-stabilized ABA triblock copolymers that possess similar thicknesses to natural lipid membranes ( $\sim 4 \mathrm{~nm}, \sim 5000$ daltons) which allowed for transmembrane water transport and constant replenishing of the electron source for release by COX (figure 1(A)). In addition, several studies have confirmed that membrane protein functionality is retained within these membranes while allowing the proteins to withstand low $\mathrm{pH}$ environments $[19,21]$. They also possess the added advantages of customizable block compositions and block lengths based upon desired applications. The overall systemic fabrication scheme served as a key component to the realization of nanoscale vesicular reactors with electrical output capabilities driven by coupled protein functionality towards applications in biosolar technology.

Bacteriorhodopsin (BR) serves as the light-dependent element of the system by aiding in the development of electrochemical proton gradients. These gradients, in addition to a heavy cytochrome $c$ mediator concentration, are used to partially reverse the activity of cytochrome $c$ oxidase (COX). This induced reaction was previously demonstrated to reverse the process of electron donation from cytochrome $c$ to COX, and instead, results in the coupling of proton translocation (gradient generated by BR) to the release of electrons that have been transferred along an iron/copper pathway in the heme-based centre of the protein (figure 1 (B) [22-26]). These electrons can then be donated to the cytochrome $c$ mediator for harvesting at electrodes. The functionalization of the block copolymer materials with these biomolecules then offers the opportunity for light-dependent direct current measurement based on protein-driven energy transduction.

\section{Methodology}

\subsection{Protein purification}

Bacteriorhodopsin from Halobacterium halobium was directly inoculated into nutrient medium and grown to a final volume of 101 at $40^{\circ} \mathrm{C}$ and $250 \mathrm{rpm}$. This culture broth was spun down in a Beckman JLA 9.1 rotor at $8000 \mathrm{rpm}$ for $30 \mathrm{~min}$ to produce cell pellets. Pellets were re-suspended in $4.2 \mathrm{mM} \mathrm{MgSO}_{4}$ solution with $0.1 \mathrm{mg} \mathrm{ml}^{-1}$ DNase I and stirred for $24 \mathrm{~h}$ at 
(A)

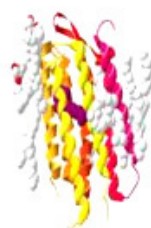

Light-Dependent Element Bacteriorhodopsin (BR)

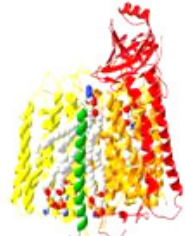

Electron-Generating Elemen Cytochrome C Oxidase (COX)
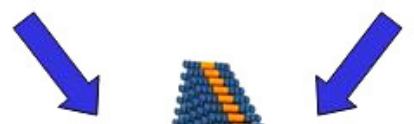
Protein Reconstitution Matrix
ABA Triblock Polymeric Biomembranes

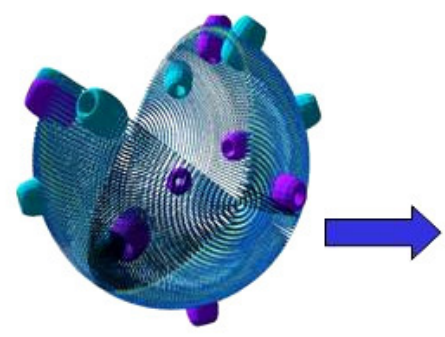

Protein-Functionalized Composite Fully-assembled vesicle
Cytochrome $\mathrm{C}_{\mathrm{rad}}(2+)$

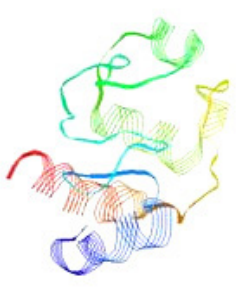

Cytochrome $\mathrm{C}_{\mathrm{ot}}\left(3^{+}\right)$

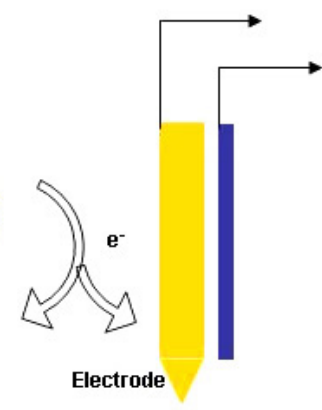

Electrode

(B)

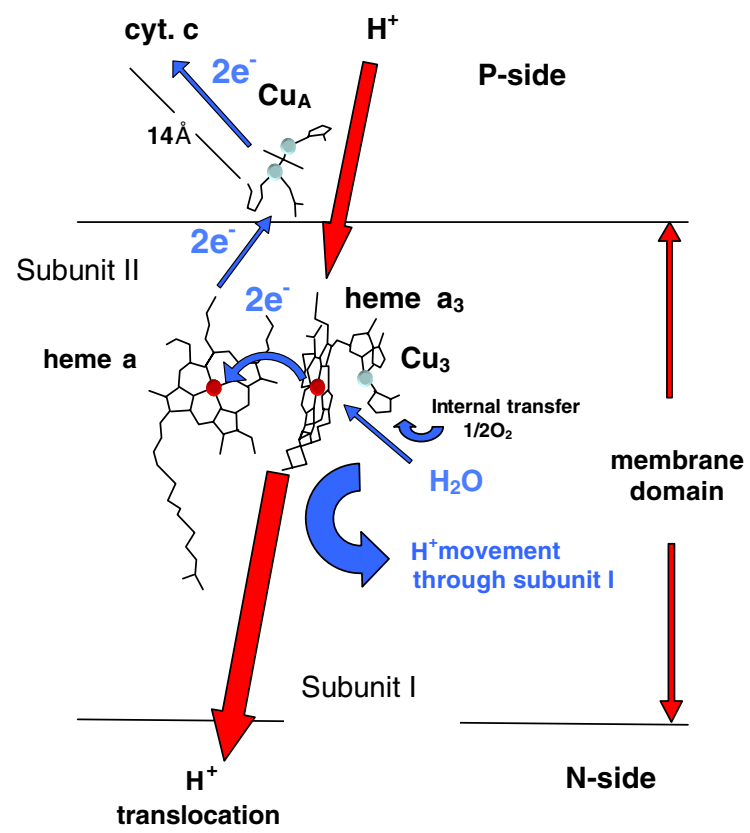

Figure 1. (A) Fabrication scheme of composite vesicles (BR/COX/cytochrome $c$ ). A depiction of the vesicle assembly is shown here as described in $[6,19]$. Bacteriorhodopsin (BR) is the element that confers light-dependence upon the system. Cytochrome $c$ oxidase (COX) is the electron-producing element. The polymer membrane serves as a matrix for protein reconstitution and facilitates oxidation-reduction reactions between the protein, the cytochrome $c$ mediator, and the electrodes. (B) Internal electron transport mechanism in cytochrome $c$ oxidase (COX). The proposed partial reversal of the electron transport mechanism of the system from [37] is depicted here, with electron movement within the heme/copper elements being coupled with the translocation of protons that are obtained from electrochemical gradients developed by BR. Though the system is depicted using two electrons, all stoichiometries should be multiplied by 2 to demonstrate oxidation of one water molecule $\left(\mathrm{O}_{2}\right)[26]$.

room temperature. Afterwards, the solution was spun down at $3000 \times g$ for 30 min in a Beckman JA 30.50 rotor, and the pellet was discarded. The supernatant was saved and centrifuged for 35 min using the Beckman Ti70 rotor at $37000 \mathrm{rpm}$. The pellet was saved and re-suspended in $\mathrm{H}_{2} \mathrm{O}$ (Nanopure). The solution was centrifuged in the Beckman Ti70 rotor at
$37000 \mathrm{rpm}$ for $30 \mathrm{~min}$. The final pellet was re-suspended with $25 \mathrm{mM}$ phosphate buffer solution containing $1 \%(\mathrm{w} / \mathrm{w})$ of Triton X-100. The solution was centrifuged in the Ti70 rotor at $37000 \mathrm{rpm}$. The supernatant was saved and injected into a HiPrep Sephacryl-100 column preequilibrated with $25 \mathrm{mM}$ phosphate buffer solution containing $1 \%(\mathrm{w} / \mathrm{w})$ of Triton X- 
100. Protein was collected in $2 \mathrm{ml}$ fractions and concentrated as desired. Purification was confirmed using SDS-PAGE gel electrophoresis.

Rhodobacter sphaeroides was used as the over expression system in the form of a genetically engineered plasmid $(15.9 \mathrm{~kb})$ for cytochrome $c$ oxidase as well as its own circular chromosomes ( $>911 \mathrm{~kb},<2973 \mathrm{~kb}$ ) [8]. The genes for the three subunits of COX (molecular masses 62.6, 32.9 and 30.1) from Rhodobacter sphaeroides are located in two separate operons along with two genes that code for proteins involved in heme $a$ synthesis. In this study, we used two different strains of Rhodobacter sphaeroides: YZ100 and 37-2. YZ100 produced COX that carried a six-residue long histidine tag at the $\mathrm{C}$ terminus of the subunit 1 , while 37-2 has enzymes which have histidine tags at the subunit 2 . The histidine tags were the key element used for nickel NTA metal affinity purification since histidine binds with nickel. For the recombinant Rhodobacter sphaeroides, the $\mathrm{pH}$ of Sistrom's media was sterilized and careful antiseptic methods of inoculation and handling were observed to minimize contamination from other microbes. Purification was confirmed using SDS-PAGE gel electrophoresis [8].

\subsection{Cytochrome c oxidation}

$30 \mathrm{mg}$ of cytochrome $c$ (horse heart muscle, Sigma-Aldrich) was dissolved in $246.2 \mu \mathrm{l}$ of water (Nanopure). A $1 \mathrm{mg} \mathrm{ml}^{-1}$ solution of ferricyanide (Sigma) was then added to the cytochrome $c$ solution. This composite solution was then added to a desalting column (Amersham Biosciences) to complete the process to produce $\mathrm{CytC}^{3+}$.

\subsection{Functionalized nanovesicle formation}

Nanovesicles have previously been shown to successfully support protein refolding and functionality. The simplified vesicle formation protocol was utilized as described in [21]. Proteins were then added to the thoroughly stirred polymer solution over several hours in order to induce integration between the proteins and polymer blocks $\left(3 \mathrm{mg} \mathrm{ml}^{-1} \mathrm{BR}\right.$ and $\left.1 \mathrm{mg} \mathrm{ml}^{-1} \mathrm{COX}\right)$. Additions were performed as described in [27] using amber vials in order to prevent BR activation. The samples were then UV-crosslinked in order to minimize vesicle permeability and enhance functionality. A $0.07 \mathrm{M} \mathrm{HCl}$ solution was then used to titrate the samples down to a starting $\mathrm{pH}$ of 3.0 to charge the solution to bring the external $\mathrm{pH}$ of the vesicles closer to the COX threshold for reversal, with the BR acting as the switch to reach threshold values. Finally, oxidized cytochrome $c$ was added as a mediator. TEM imaging $(100000 \times)$ was used to confirm vesicle formation.

\subsection{Current measurement from composite (BR/COX/cytochrome c) vesicles}

Electrical measurements were obtained using a standard electrochemical direct current measurement as described in $[6,21]$ using the precision semiconductor parameter analyser (Agilent) as described. Electrodes were attached to an Agilent test fixture via miniature banana-to-clip probes (Agilent). The working electrode was significantly larger in diameter (minimum $5 \times$ ) than the reference electrode to facilitate unidirectional electron transfer. Data were sampled at approximately $12 \mathrm{~Hz}$ for $50 \mathrm{~s}$ at a time with and without illumination. After equilibration, the samples were read in alternating light/dark sequences, each with $50 \mathrm{~s}$ exposures. Experiments were performed to measure the composite vesicles as an anodic electron-generating process of a half cell, whereby the electrons can then travel to the load and cathode. Future experiments will consider the fabrication of the complete dual cell energy system.

A microfabricated three-Au-electrode system (Genefluidics) was used for measurement of protein-induced oxidationreduction at the electrode surface for cyclic voltammetry measurements. Measurements were done using sweep mode with current plotted versus time. All tests were run using a $150 \mathrm{~W}$ lamp (Fostec) for illuminations. CV scan parameters included a range of -0.5 to $0.5 \mathrm{~V}$ at a scan rate of $0.01 \mathrm{~V} \mathrm{~s}^{-1}$ and a sample interval of $0.001 \mathrm{~V}$.

\subsection{Measurement of current output from COX-functionalized vesicles}

COX-functionalized vesicles were prepared as previously described. The electrical measurement setup was also previously described. $0.07 \mathrm{M} \mathrm{HCl}$ was added dropwise to the solution and measured over the course of $10 \mathrm{~min}$. Current versus time plots were generated to correlate current increases with the change in $\mathrm{pH}$ level.

\subsection{Measurement of bacteriorhodopsin-induced pH change}

The proton pumping activity of the purple membrane in the polymer vesicle was optically monitored using the 8-hydroxyprene-1,3,6-trisulfonic acid fluorescence probe, pyranine. The excitation scan by luminescence spectrometer (Perkin Elmer) was performed in the range of 350-475 nm at an emission of $511 \mathrm{~nm}$. External pyranine was quenched by the addition of $p$-xylenebispyridinium bromide (DPX) to a final concentration of $20 \mathrm{mM}$. The conversion from the fluorescence to $\mathrm{pH}$ was performed as described in [28].

\subsection{UV-vis measurement of water transport through polymer membranes}

Polymer vesicles were fabricated as previously described and syringe filtered to provide a uniform formation of $250 \mathrm{~nm}$ diameter vesicles. The external solute concentration to the vesicles was increased by adding $0.2 \mathrm{M} \mathrm{KCl}$. The gradual change in absorbance believed to be attributed to water transport out of the vesicle was observed using UVvis spectrophotometry. Samples measured included bufferonly, vesicle solution in the dark for several minutes to assess absorbance drift in the spectrophotometer (minimal), as well as the vesicle solution with the external $\mathrm{KCl}$ gradient.

\section{Results and discussion}

\subsection{Block copolymer characterization}

Our integrated biologically active thin films consisted of BR and COX inserted into block copolymeric membranes that simulate the environments found in conven- 


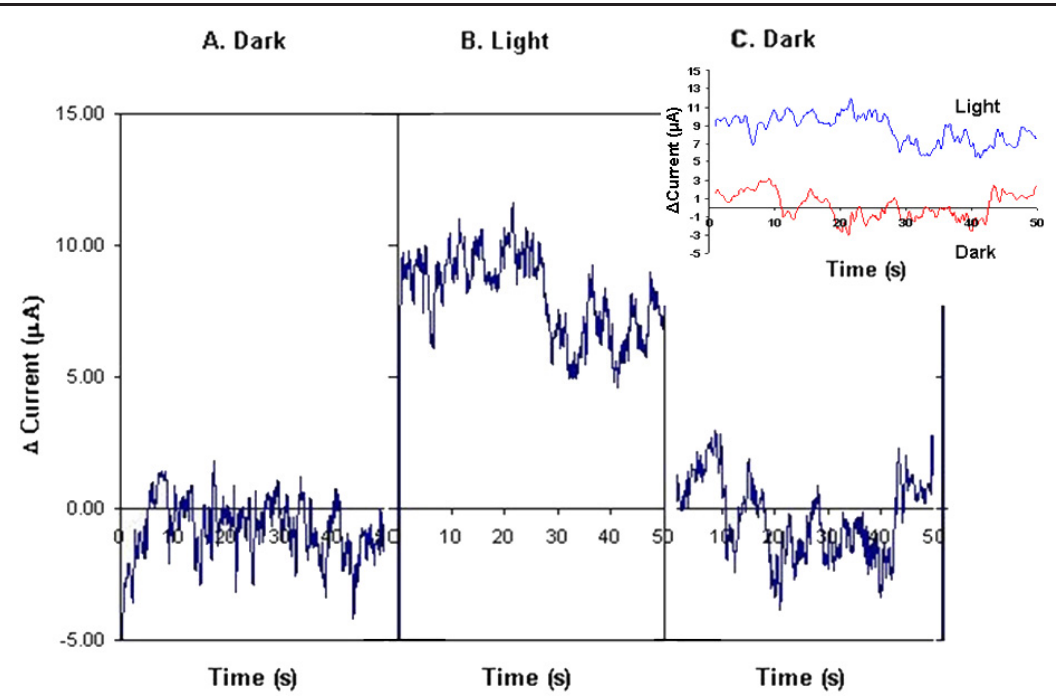

Figure 2. Alternate cycles of current switching. Steady current generation from composite vesicles samples was measured using $50 \mathrm{~s}$ intervals of light/dark environments. Current versus time measurements were taken to observe coupled functionality without applied voltages. Incubation of the samples with light resulted in current increases as high as $10 \mu \mathrm{A}$ compared with samples scanned in the dark. The stochastic mechanisms of vectorial ion transport in the BR protein and transient $\mathrm{P}-\mathrm{F}$ transition of COX proteins due to the decay of the electrochemical proton gradient are phenomena that have been well studied and were commonly expected occurrences within the system. This may have played a role in the spontaneous inactivation/reactivation of proteins during current generation. $\left(I_{\text {mean }}=10 \mu \mathrm{A} ; \mathrm{SD}=0.28\right.$; $n=6$; uncertainty relative $=2.8 \%$.) Inset: the measurement of steady currents in 'on' and 'off' states is illustrated here as seen by the increases and decreases in current output depending on the environment of the composite vesicles with an approximate maximum amplitude of $10 \mu \mathrm{A}$.

tional lipid systems. An ABA triblock copolymer consisting of polymethyloxazoline- $b$-polydimethylsiloxane- $b$ polymethylozaxoline (PMOXA-PDMS-PMOXA) was fabricated to a thickness of approximately $4 \mathrm{~nm}$ to mimic naturally occurring biomembranes. Thicknesses were determined using irreversible rupture tests whereby the biopolymer film capacitance generated while suspended across a hydrophobic septum was used to calculate the film thickness as given by

$$
C=\frac{\varepsilon_{0} \varepsilon_{1} A}{d},
$$

where $C$ is the membrane capacitance, $\varepsilon_{0}$ is the permittivity of free space $\left(=8.9 \times 10^{-19} \mathrm{~F} \mathrm{~m}^{-1}\right), \varepsilon_{1}$ is the relative dielectric constant of the PDMS hydrophobic block, $A$ is the measured area of the annulus in the septum $(=250 \mu \mathrm{m})$, and $d$ is the thickness of the membrane [13-15]. To test for enhanced membrane durability over conventional lipid systems, membrane rupture tests were conducted utilizing dual $\mathrm{Ag} / \mathrm{AgCl}$ electrodes on both sides of the suspended polymer membrane. Transmembrane voltages of increasing magnitude were applied to observe electric-field-induced irreversible rupture associated with termination of the membrane. It was observed that the UV-crosslinked polymer membranes could withstand transmembrane voltages as high as $1.5 \mathrm{~V}$ (uncrosslinked $=\sim 1 \mathrm{~V}$ ) compared with lipid systems that commonly ruptured at $500 \mathrm{mV}$, indicating an enhanced mechanical stability in the polymer membranes [13]. In addition, Langmuir isotherms were conducted to compress the copolymer films to measure film collapse pressures. We have previously shown that the polymer collapse pressures, in excess of $70 \mathrm{mN} \mathrm{m}{ }^{-1}$, were significantly higher than those observed in lipid systems $\left(\sim 50 \mathrm{mN} \mathrm{m}^{-1}\right)$ [29]. With both the irreversible rupture and Langmuir collapse studies, increased polymeric mechanical strength over lipids was observed, as was the fact that the polymers could withstand higher Langmuir surface compression pressures. As such, the biomimetic properties and robustness of the PMOXA-PDMS-PMOXA polymer make it an ideal engineering component for biosolar films.

\subsection{Composite vesicle direct current measurement}

Exposure of the composite proteopolymersomes (BR/COX/ cytochrome $c$ ) to green light with $50 \mathrm{~s}$ durations resulted in current ${ }_{\text {on }}-$ current $_{\text {off }}$ versus time plots as high as $10 \mu \mathrm{A}$ compared with initial measurements when the vesicles were exposed to 'dark' environments (figure 2). Subsequent exposure of the proteopolymersomes with 'dark' conditions resulted in a switching of the current back to a lower current value. This cycle of on/off switching could be accomplished approximately ten times before differences in current output for the on/off states were no longer observed. Samples without BR that were illuminated did not produce a current change and possessed no light-dependent activity. An estimation of device efficiency in the visible spectrum (350-750 nm) offered by the source was determined by comparing the measured $3.4 \mathrm{~W} \mathrm{~m}^{-2}$ of the active area (based on maximum current value $=\sim 10 \mu \mathrm{A}$; power $=\sim 2.4 \mu \mathrm{W}$ based on measured/observed cytochrome $c$ electron transfer potential of $0.235 \mathrm{~V}[30,31]$; and area of $0.00694 \mathrm{~cm}^{2}$ ) to the LUX (lumens/square metre) obtained from the light source. This value was obtained by utilizing a light intensity that was $1 / 25$ of that provided by average sunlight $\left(1350 \mathrm{~W} \mathrm{~m}^{-2}\right)$, resulting in $54 \mathrm{~W} \mathrm{~m}^{-2}$. A comparison of both values would result in an assayed maximum power $r_{\text {out }} /$ power $_{\text {in }}$ efficiency of $6 \%$ which also corresponds to a current density of $0.14 \mathrm{~mA} \mathrm{~cm}^{-2}$. It 
Fabrication of biomolecule-copolymer hybrid nanovesicles as energy conversion systems

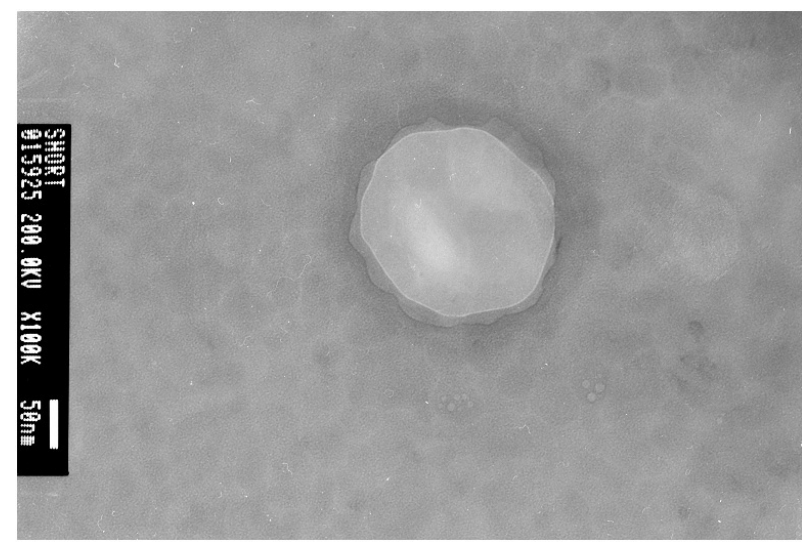

Figure 3. Transmission electron microscopy (TEM) imagery of polymer vesicle. A $100000 \times$ image of a polymer vesicle is shown here possessing a diameter of $\sim 200 \mathrm{~nm}$. Vesicles were not readily visible via TEM until syringe filtration was utilized. It was believed that the filtration process removed multilamellar structures.

should be mentioned that given that there was not a precise method of uniform reconstitution of functional COX (electrongenerating species), varying $\mu \mathrm{A}$-range current outputs were observed. As such, the calculated power ${ }_{\text {out }} /$ power $_{\text {in }}$ efficiency was based on the current $t_{\max }$ observed. Optimization studies will attempt to enhance abilities to reconstitute a majority of the injected COX protein as the polymeric membrane has been previously demonstrated to be extremely cohesive, which may have led to a tendency for protein exclusion [13], although studies have also documented that it is still energetically favourable for these polymeric spheres to house reconstituted proteins while facilitating their functionality [32]. Additional reasons for these observed current output variations are also based on the fact that the methodology used to fabricate the composite vesicles does not possess the ability to control the number of functional vesicles formed. For example, vesicle formation was usually not visible until after syringe filtration of the bulk vesicle/protein solution (figure 3). Filtration was used to remove multilamellar structures that were not functional or to form uniform unilamellar structures. In addition, the process of forming the bulk polymer/protein solution would have certainly resulted in inserting improperly oriented or refolded proteins.

\subsection{Composite vesicle cyclic voltammetry and cytochrome $c$ analysis}

It is well known and has been previously demonstrated that cytochrome $c$ in solution serves as an effective means of electron delivery to the electrode, demonstrating its importance in the functionality of the system [33-35]. As measurements were being taken in solution with free-floating composite proteopolymersomes that were not directly adsorbed to the electrode, we believed electron transfer to the electrode to be mediated by cytochrome $c$, demonstrated by the fact that removal of the mediator resulted in a cessation of reduction at the electrode (figure 4 , inset). We have previously measured composite vesicle current output using cyclic voltammetry, and compared this output with blank electrode readings as

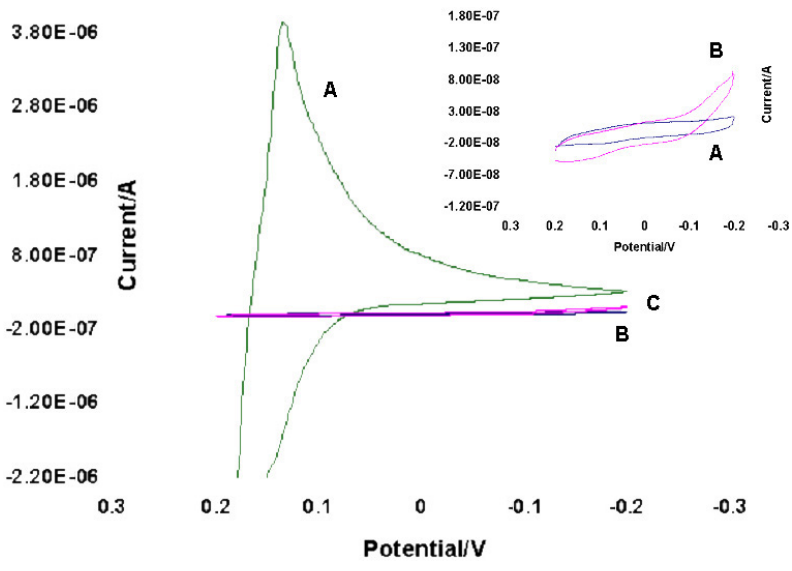

Figure 4. Cyclic voltammetry of protein-functionalized vesicles. A comparison between oxidation/reduction activity of protein-functionalized vesicles (A), and buffer-only blank electrode (B) as well as oxidized cytochrome $c$ species (C) is observed here. The mediator is re-oxidized so that it is prepared to receive electrons from the COX protein. It was observed that a significantly larger current output was observed for the protein-functionalized samples compared with the blank electrode as well as cytochrome $c$-only readings. Current output from the functionalized vesicles (A) was obtained from [46]. Inset: a cyclic voltammetry cytochrome reading of blank electrode (A) versus oxidized cytochrome $c$ (B) shows an absence of the pronounced redox reactions that are observed with protein-functionalized samples.

well as solutions containing only oxidized cytochrome $c$ [36]. Figure 4 shows that the protein-functionalized polymers were capable of producing current outputs that were significantly greater than those of the blank and cytochrome $c$ electrode reactions. In addition, it was shown that a variable current switching could be observed between light (on) and dark (off) states for the protein-functionalized vesicles [36]. Also, it was demonstrated that the sole addition of oxidized cytochrome $c$ did not result in an electrode surface reaction, which was also observed in the blank electrode arrangement [36]. These observations were expected as oxidized cytochrome $c$ as well as the blank buffer did not contain bound electrons that could be used to reduce the working electrode. However, composite vesicle solutions in the presence of illumination resulted in the observed redox reactions at the electrode which was an indicator of coupled protein functionality (figure 4). Illumination of the blank electrode and cytochrome $c$ samples resulted in no current generation to eliminate the light as an indirect cause of redox reactions at the electrodes. In addition, shifts in observed redox potentials have been previously attributed to factors including varying types of metals being used for the working, counter, and reference electrodes, as well as varying oxygenation levels of samples [33]. In fact, formal potentials ranging from 143 to $250 \mathrm{mV}$ have been observed due to these factors [37]. Any instances of observed irreversibility of reaction were attributed to extraneous chemical reactions that were believed to include adsorption of composite vesicle systems or cytochrome $c$ mediators that were in an oxidized state (Ox), after donating electrons to the cytochrome $c$ or the gold working electrode, respectively. Even though the cytochrome $c$ electron transfer properties are known and characterized as being rapid and reversible, in this case, 
adsorption would have disrupted the electron transfer off of the electrode, rendering the redox reaction as irreversible, which is in agreement with the literature [38].

Because heme groups can transfer electrons over distances of up to $14 \AA$ at significant rates, cytochrome $c$ is able to exchange electrons at a very high rate. The heterogeneous reaction of a redox species at an electrode is given by

$$
A_{\mathrm{O}}^{n+}+B_{\mathrm{R}} \rightarrow A_{\mathrm{R}}+B_{\mathrm{O}}^{n+}
$$

where $n$ electrons are transferred from reductant $\left(B_{\mathrm{R}}\right)$ to oxidant $\left(A_{\mathrm{O}}\right)$. The free energy of this reaction is the work required to move electrons through a potential difference $\Delta E$ and is given by the following equations:

$$
\begin{gathered}
\Delta G=-n F \Delta E \\
\Delta G=\Delta G^{0}+R T \ln \left(\frac{C_{A_{\mathrm{R}}} C_{B_{\mathrm{O}}^{n+}}}{C_{A_{\mathrm{O}}^{n+}} C_{B_{\mathrm{R}}}}\right)
\end{gathered}
$$

where $C_{i}$ are the concentrations of species $i, R$ is the universal gas constant, $T$ is temperature, and $F$ is Faraday's constant (96 $494 \mathrm{C} \mathrm{mol}^{-1}$ ).

The electric potential is given by

$$
\Delta E=\Delta E^{0}-\frac{R T}{n F} \ln \left(\frac{C_{A_{\mathrm{R}}} C_{B_{\mathrm{O}}^{n+}}}{C_{A_{\mathrm{O}}^{n+}} C_{B_{\mathrm{R}}}}\right) .
$$

If we consider only one redox pair, we have

$$
E=E^{0}-\frac{R T}{n F} \ln \left(\frac{C_{\mathrm{R}}}{C_{\mathrm{O}}}\right) .
$$

If the reaction given by equation (2) is fast and theoretically reversible, the solution near the electrode will be at equilibrium and the redox pair and electrode will be at the same potential. If the equilibrium is perturbed, producing an increase in concentration of either the reductant $\left(C_{R}\right)$ or oxidant $\left(C_{\mathrm{o}}\right)$, the potential of the electrode and solution will no longer be in equilibrium and the potential will decrease or increase, respectively, as governed by equation (5). However, electron exchange between cytochrome $c$ and the electrode has been observed to be fast enough so that this potential difference is small [33-35]. Furthermore, because cytochrome $c$ closely interacts with $\mathrm{COX}$, electron transfer between cytochrome $c$ and $\mathrm{COX}$ also occurs at significant rates, making cytochrome $c$ a fast and efficient electron mediator between the electrode and COX [20].

\subsection{Analysis of BR-dependent gradient generation}

BR functionality in polymeric membranes has previously been demonstrated, which in turn conferred the lightdependent aspect into the composite proteopolymersomes [6]. Furthermore, the artificially induced $\mathrm{pH}$ gradient using a $0.07 \mathrm{M} \mathrm{HCl}$ solution (pre-threshold value of $\Delta \mathrm{pH}=4.0-4.2$ ) in the external vesicle environment served to bring the medium to a point right before the threshold for $\mathrm{COX}$ reversal, allowing $\mathrm{BR}$ to serve as the light-dependent switch. Measurement of proton pumping by $\mathrm{BR}$ was performed by enclosing pyranine, a pH-sensitive dye, within vesicles and measuring the fluorometric $\mathrm{pH}$ change (Perkin Elmer). It was also observed

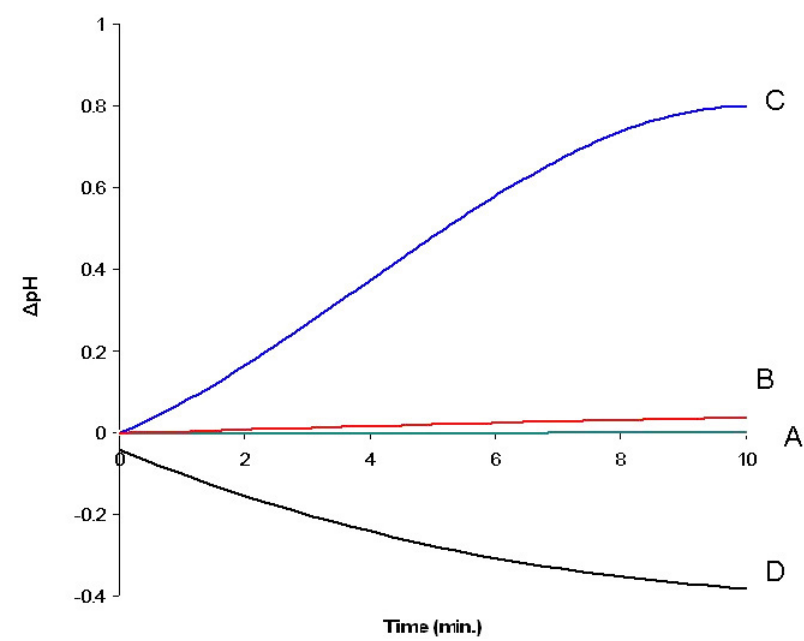

Figure 5. Protein-induced $\mathrm{pH}$ change comparison/physiological orientation of BR. The increase in achievable $\mathrm{pH}$ changes across the membrane as a result of BR activity is shown here. When the BR protein is absent from the vesicles, no light-dependent $\mathrm{pH}$ change is observed (A), indicating that BR is the light-dependent element of the system. The $\mathrm{pH}$ change from $\mathrm{BR}$ vesicles without crosslinking (B) can be increased once the vesicles are crosslinked (C). When proteins are reconstituted in low $\mathrm{pH}$ environments, it has been shown that physiological orientation or proton pumping out of the vesicle can be achieved [31] (D). This is shown by the negative $\mathrm{pH}$ change, or net proton removal from the interior of the vesicle. In addition it should be noted that the magnitude of $\mathrm{pH}$ change $(\Delta \mathrm{pH}=4.2$, solution $\mathrm{pH} \sim 3.0-3.2)$ is already against a pre-addition of $0.07 \mathrm{M} \mathrm{HCl}$ solution that is already near the threshold for COX reversal and the BR serves as a switch to utilize illumination to reach threshold for COX partial reversal.

that $\mathrm{pH}$ changes could be increased by crosslinking the polymer membrane using shortwave ( $254 \mathrm{~nm}$ ) UV light (figure 5). This was believed to increase protein functionality due to increased mechanical stability of the membrane as well as decreased shielding of the BR from light [19, 21]. Furthermore, upon the incubation of BR-functionalized proteopolymersomes with green light in low surrounding $\mathrm{pH}$ conditions (3.0), it was observed that the $\mathrm{pH}$ level within the polymeric vesicle increased, indicating a net outward flow of protons due to an achieved 'physiological' orientation of the protein (figure 5). This $\mathrm{pH}$-induced orientation of BR has been previously shown to be due to a modified surface polarity of the membrane that favours the physiological orientation of the protein [19, 39] This orientation is noted in the negative $\mathrm{pH}$ change with respects to the internal $\mathrm{pH}$ of the vesicle, signalling an outward flow of protons. These protons can then travel back into the vesicle to effect electron transfer to the exterior of the vesicle and delivery to the cytochrome $c$ mediator.

Current versus time plots for samples containing only COX and only cytochrome $c$ resulted in no changes in current These observations eliminated the light source as a cause for current increases. The coupling of BR removal with loss of current switching was also an indication that it was the light-dependent element of the composite vesicle system given by the loss of proton pumping activity observed in the polymer membrane using pyranine (figure 5). In addition, UV-vis spectrophotometric analysis of the composite vesicles demonstrated that chromophoric activity of the BR light- 


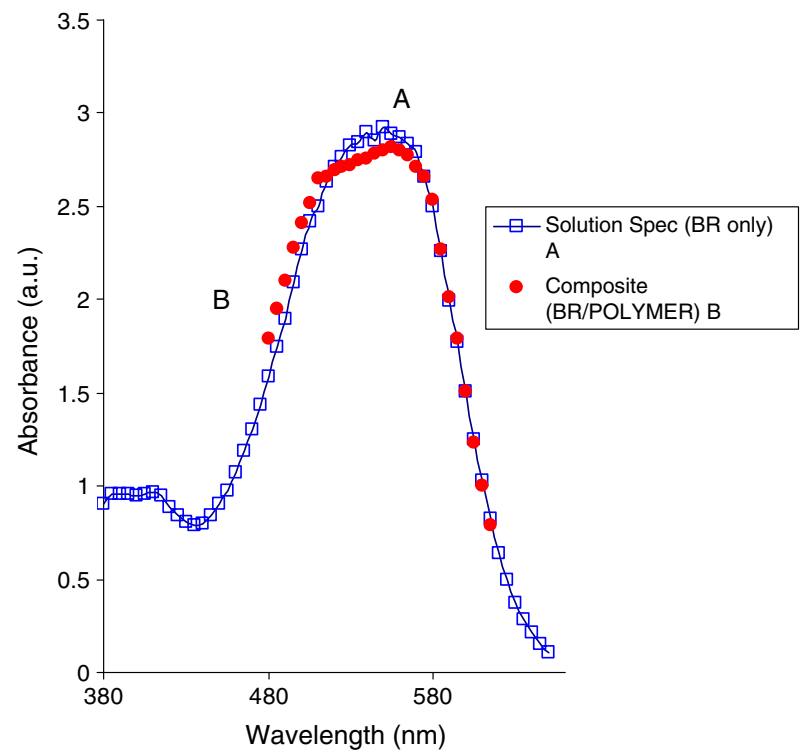

Figure 6. UV-vis spectrophotometric measurement of retinal viability. In addition to BR-driven $\mathrm{pH}$ change, UV-vis spectrophotometry was used to measure the spectral properties of $\mathrm{BR}$ in solution versus a BR/polymer mixture. BR spectral activity was shown to be preserved at $563 \mathrm{~nm}$. Furthermore, it was observed that the polymer could maintain retinal viability in very low $\mathrm{pH}$ conditions $(<2)$ while the lipid solutions could not maintain chromophore viability in these environments.

reactive retinal element at $563 \mathrm{~nm}$ was preserved in the sample, indicating that retinal was viable (figure 6).

The mechanism of unidirectional transport has been well characterized, both structurally and mechanistically, through several methods including FTIR spectroscopy, ESR spectroscopy, and laser spectroscopy [40-43]. A foundational and basic description of the vectorial transport process can be assessed using three processes, denoted as I (isomerization), $\mathrm{T}$ (ion transport), and $\mathrm{S}$ (accessibility change). The vectorial catalysis of proton transport begins with the conformational change of the retinal chromophore (covalently bound via Schiff base to K126 on helix G) from an all-trans to a 13-cis state. Subsequently, an increase in strength of the hydrogen bonding interaction between the Schiff base and D85 is accomplished via a water molecule, which is followed by proton transfer from the Schiff base to the D85 in the extracellular region. Because BR-driven proton transport is a unidirectional, vectorial process, deprotonation of the Schiff base occurs in conjunction with the non-accessibility of the Schiff base to reprotonation by D96. After D96 is reprotonated on the intracellular side, the Schiff base points towards the extracellular side to re-initiate the starting point of vectorial catalysis [66].

It should be mentioned that while the currents generated by protein coupling were relatively steady, the transient shifts in current output could be attributed to spontaneous protein inactivation and reactivation. The stochastic nature of vectorial ion transport in the BR protein as well as transient shut-off of COX proteins due to the decay of the electrochemical proton gradient are phenomena that have been well studied and were commonly expected occurrences within the system.
A multitude of studies have investigated the modelling of BRdriven vectorial ion transport. For example, basic descriptions have explained the enzymatic reaction process as a single-ion transport step [44]. In several cases, ATP-utilizing pumps as well as redox pumps have all been characterized as having two conformational states $[45,46]$. A stochastic energizationrelaxation model has also been applied specifically to BR and was based upon the derivation of an external energy supply from photoreactions. This model states the ion transport was driven by asymmetrical potential profiles along the transport path, that the coupling between ion transport and conformational changes was stochastic, and that the vectorial catalysis rate was dependent on ion concentration gradient and membrane potential which was in agreement with existing studies [47]. These comprehensive mechanistic and theoretical studies, coupled with numerous experimental investigations, have provided a firm foundation supporting the vectorial catalysis process in BR.

\subsection{Assessment of COX transitional states}

In addition to observing fully assembled vesicle activity, it was important to determine that the current generation could have been in fact due to partial COX reversal. Experimental results showed that vesicles functionalized with only BR, only COX, or vesicles without proteins were all unable to produce the light-dependent current increases. As previously shown, the light-dependent current generation could only be achieved upon the addition of both BR and COX to the vesicles. Furthermore, we have previously shown that BR is capable of pumping out about $1.44 \times 10^{14}$ protons, correlating to approximately $6.2 \times 10^{9}$ active protein monomers in the film, which is substantially below the amount of protein added to the solution [6]. After previously determining that the lightdependent element was due to BR proton pumping activity, the electrical element contributions from COX due to its electrochemical gradient-induced partial reversal was tested by measuring the current output from vesicles that contained only COX. Using a $0.07 \mathrm{M}$ hydrochloric acid $(\mathrm{HCl})$ solution as a simulation for BR activity, we slowly titrated the surrounding medium of the COX vesicles and measured the $\mathrm{pH}$ change against current production occurrences. A high concentration of oxidized cytochrome $c$ was also added to serve as the electron acceptor, as well as to contribute to the driving force to reverse COX. This high concentration of cytochrome $c$ (determined to be $>25 \mu \mathrm{M}$ in [48]) while poised at a high redox potential $\left(E_{\mathrm{h}}\right)$ served to enable its capacity as an electron acceptor enhance interaction between the cytochrome $c$ and the COX. By applying the external electrochemical proton gradient to the COX vesicles, an increase in current was observed (figure 7). Because there was no BR inserted into the membranes, the electrochemical gradient could not be regenerated by BR pumping. As such, we expected to observe only a single current increase occurrence due to complete cessation of COX reversal. The current increase occurred with the $\mathrm{pH}$ change of $\sim 4.5$, accounting for drift from real-time $\mathrm{pH}$ acquisition, error limits in the $\mathrm{pH}$ probe, and transmembrane ion flux.

Based upon an oxidation-reduction potential for cytochrome $c$ of $235 \mathrm{mV}$ as described in [30], high 


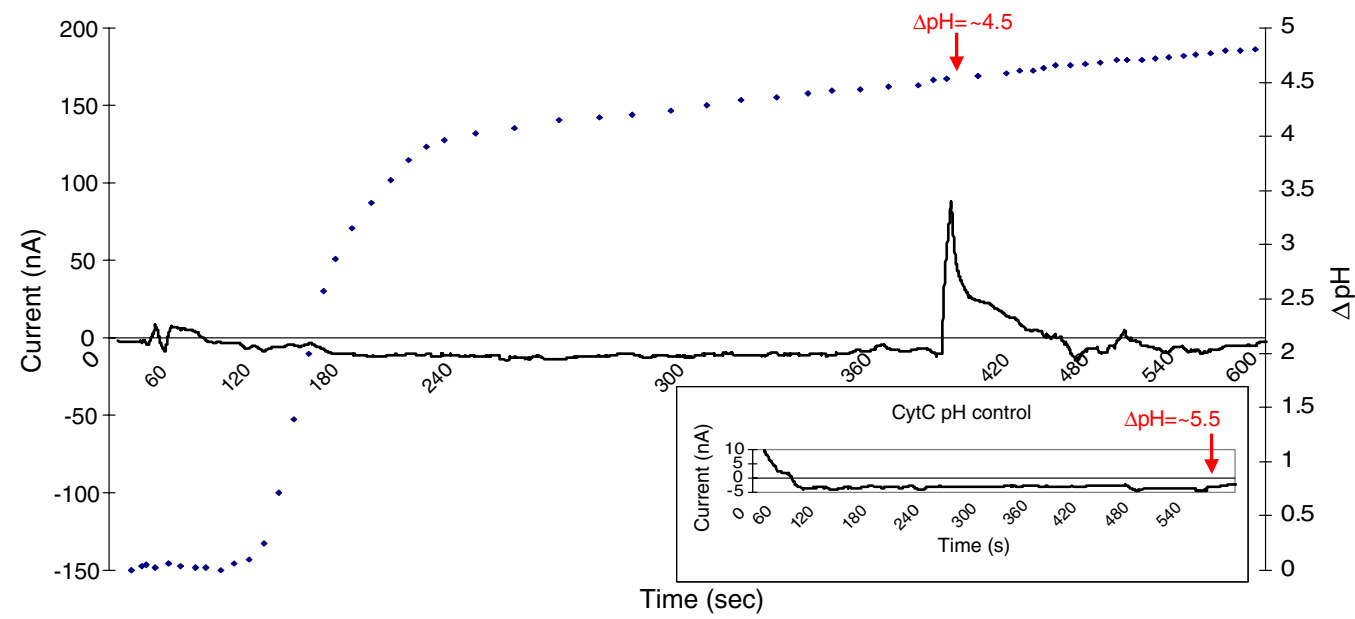

Figure 7. pH-dependent COX oxidation/cytochrome $c$ reduction. The addition of $0.07 \mathrm{M} \mathrm{HCl}$ to COX-functionalized vesicles and a high concentration of oxidized cytochrome $c$ resulted in current production at $\sim \Delta \mathrm{pH}=4.5$. This was comparable to estimations of $\sim \Delta \mathrm{pH}=4$. Inset: vesicles that did not contain the COX protein resulted in an absence of current increases after a $\Delta \mathrm{pH}$ of at least 5.5 units.

concentration of oxidized cytochrome $c^{3+}(>25 \mu \mathrm{M})$, as well as room temperature conditions, it was previously postulated that the partial reversal of COX could be achieved at a pH level of at least 4 units [20, 48-50]. These parameters would have provided the adequate driving force to work against the energy barrier of water oxidation [50]. Controls were performed by adding the electrochemical proton gradient to samples containing cytochrome $c$ and polymer in the analyte buffer with no protein. This resulted in no current being generated through a $\mathrm{pH}$ range of at least 5.5 units.

In addition to direct current measurement, UV-vis spectral analysis of the COX reversal process was conducted, and resulted in the gradual increase in absorbance of the COX $630 \mathrm{~nm}$ peak based upon the continual addition of $\mathrm{HCl}$ to the vesicle solution, which was previously shown in the literature to characterize the partial reversal of the COX pathway (figure 8) [48]. The disappearance of the oxidized heme at $550 \mathrm{~nm}$ and the appearance of the $630 \mathrm{~nm}$ absorbance peak indicated that there was a shift in COX intermediates $(\mathrm{F}-\mathrm{P})$ that had been reached [22, 49]. The $630 \mathrm{~nm}$ species was defined previously as a peroxy intermediate, or P state (ferryl heme/cupric-tyrosine radical), and the $550 \mathrm{~nm}$ species as the $\mathrm{F}$ state intermediate (ferryl/cupric-dioxygen). The analysis of the spectral properties of the COX reversal intermediates can be described as the transition from state $\mathrm{O}$, which is converted to state $\mathrm{F}$, and then to the $\mathrm{P}$ intermediate, with one electron being released from each conversion process to cytochrome $c$ during the addition of the external proton source from the $\mathrm{HCl}$. Our spectral measurements of maximized COX reversal to the $\mathrm{P}$ state revealed a molar absorptivity of $25 \mathrm{mM}^{-1} \mathrm{~cm}^{-1}$ that was based upon a $10 \mathrm{~mm}$ path length, species concentration of $0.04 \mathrm{mM}$, and $\Delta A$ of $\sim 1$. This measurement was comparable to the measurement obtained in [48] of $11 \mathrm{mM}^{-1} \mathrm{~cm}^{-1}$ with variations being possibly due to varying amounts of integrated protein or discharge of the proton gradient $\Delta \mu_{\mathrm{H}}^{+}$. Previous work has also shown how the $630 \mathrm{~nm}$ spectral indication of the P intermediate can also be used to analyse P-F state interconversion depending on the

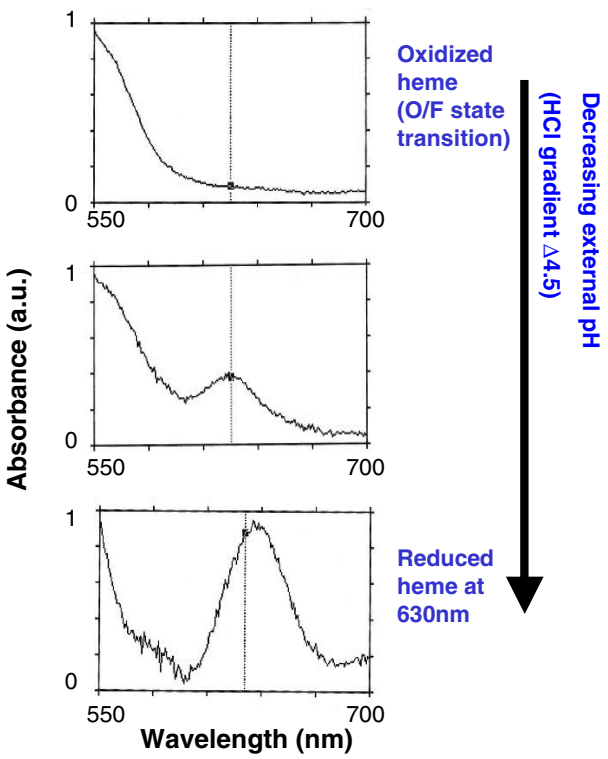

Figure 8. UV-vis measurement of cox intermediate states. The gradual shift of the spectral properties of COX from 550 to $630 \mathrm{~nm}$ indicated that the protein was able to achieve several intermediates indicative of partial COX reversal. More specifically, the disappearance of the $550 \mathrm{~nm}$ peak indicated that the COX shifted out of the $\mathrm{O}$ intermediate towards the $\mathrm{F}$ intermediate whereby an electron is released to cytochrome $c$. A shift to the P intermediate given an adequate sustaining electrochemical proton gradient and high cytochrome $c$ concentration would have resulted in a full transition to the $\mathrm{P}$ intermediate which would release another electron to cytochrome $c$.

increase/decrease in magnitude of the optical peaks (e.g. $\Delta$ absorbance) [48].

The $\mathrm{F}$ and $\mathrm{P}$ intermediates are both characterized as conditions where COX coupled proton translocation with electron release and signify the desired states to be achieved through coupling with BR. The proportions of these intermediates that are finally formed depend on the 


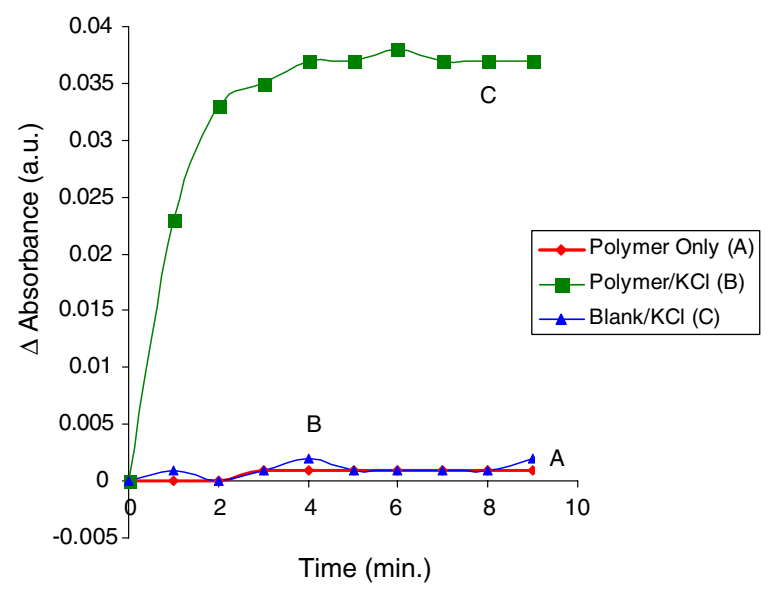

Figure 9. UV-vis measurement of transvesicular water transport. The establishment of water transport through polymer membranes has been established. The polymer was fabricated to possess a molecular weight of $~ 5000$ daltons as well as UV-reactive endgroups that could be crosslinked using $254 \mathrm{~nm}$ UV light. This architecture was believed to be an optimized blend of membrane fluidity and stability to enhance resistance to defect formation while enabling transmembrane water transport.

concentration/acceptor potential of cytochrome $c\left(E_{\mathrm{h}}\right)$, as well as the electrochemical proton gradient $\left(\Delta \mu_{\mathrm{H}}^{+}\right)$. It was previously determined that when all of these parameters are maximized, virtually all the enzyme can be converted into the $\mathrm{P}$ state $[48,49]$. This information has been confirmed by the observation that the absorption increase at $630 \mathrm{~nm}$ is dependent primarily on the $\mathrm{pH}$ gradient and can be continually increased through the addition of acid to the solution until the maximum is reached [51]. In the case of the $630 \mathrm{~nm}$ reduced peak in figure 9 , the continued addition of $\mathrm{HCl}$ to the solution resulted in cessation of absorbance growth, indicating an optimal $\mathrm{P}$ intermediate conversion.

The phenomenon of reversed electron transfer in COX was adapted from [52] and can be described thermodynamically by the following relationship:

$$
E_{2}-E_{1}=\frac{\Delta G}{n F}
$$

where $E_{2}$ and $E_{1}$ are the respective redox potentials on the high and low potential sides of the energy-conservation site, $\Delta G$ is the Gibbs free energy change in ATP production from $\mathrm{ADP}$ and $P_{i}$ in chemiosmotic systems such as the respiratory chain, which is coupled with the forward reaction of COX as a proton pump (also commonly referred to as the phosphorylation potential, and shown to be dependent upon $\Delta \mathrm{pH}$ and ATP-dependent effects on $\left.E_{2}\right), n$ is the number of electrons transferred, and $F$ is the Faraday constant [48, 53]. During the transfer of electrons from cytochrome $c$ to COX (forward reaction), the electrons are continuously decreasing their free energy, eventually reducing $\mathrm{O}_{2}$ at the luminal side of the membrane. The free energy change per electron transferred is $-14.8 \mathrm{kcal} \mathrm{mol}^{-1}$. This energy is used to transport protons to the external side and the accumulated protons create the electrochemical gradient across the membrane. Initial studies have been conducted pertaining to reversed electron transfer that were based on ATP-driven reversal in electron transport chains. As previously mentioned, the addition of ATP to electron transport chains can reverse proton-assisted ATP production to result in ATP-driven proton release. As such, the addition of excess ATP (product) to chemiosmotic systems was directly correlated to proton release (reactant) and a resultant increase in $E_{2}$ to cause the acceptor to become more oxidized and a resultant decrease in $E_{1}$ to create a reduced donor, thus also resulting in a higher $\mathrm{pH}$-dependent $\Delta G$ for the forward reaction and a reverse electron transfer process [52].

Our previously mentioned observations of proton gradient-associated $(\mathrm{HCl})$ spectral changes resulting in the transition towards a maximum $630 \mathrm{~nm} \mathrm{P}$ intermediate species are in agreement with the literature that the external proton concentration was increased sufficiently high enough (correlated with the assisted proton pumping activity of BR in our experiments, as well as a high $[\mathrm{ATP}]:[\mathrm{ADP}]\left[\mathrm{P}_{i}\right]$ ratio in chemiosmotic systems) to create a positive $\Delta G$ for the forward reaction of COX due to the dependence of $\Delta G$ on the surrounding $\mathrm{pH}$. This phenomenon is associated with being able to alter the equilibrium that is normally associated with the $\mathrm{COX}$ forward reaction of $\mathrm{O}_{2}$ reduction to water, effectively driving this forward process in reverse [22, 53]. In this case, forward pumping of protons required more energy than the reduction of $\mathrm{O}_{2}$ could provide. Therefore, when there was a sufficient concentration of hydrogen present on the external side, the COX function favoured a reversed reaction, receiving electrons from oxidized water which were eventually donated to cytochrome $c$. As the external $\mathrm{pH}$ increased, $\Delta G$ was enhanced, favouring a reversed electron transfer.

The kinetic basis of partial reversal of COX based upon the successive transition between the $\mathrm{O}, \mathrm{F}$, and $\mathrm{P}$ states of the $\mathrm{a} 3 / \mathrm{Cu}_{\mathrm{b}}-\mathrm{iron} /$ copper sites, as well as cytochrome $c$ reduction by COX, has also been demonstrated in the literature, where it was clearly observed that the $\mathrm{F}$ intermediate was formed prior to the $\mathrm{P}$ intermediate and immediately followed by a rapid reduction of the cytochrome $c$ that was introduced at a high concentration $[21,48]$. The $\mathrm{O}-\mathrm{F}-\mathrm{P}$ transition reaction could thus be given by

Reverse step 1 (O-F transition):

$$
\mathbf{O}+c^{3+}+2 \mathrm{H}_{2} \mathrm{O}+\text { energy }=\mathbf{F}+c^{2+}+2 \mathrm{H}_{\mathrm{M}}^{+} .
$$

Reverse step 2 (F-P transition):

$$
\mathbf{F}+c^{3+}+\text { energy }=\mathbf{P}+c^{2+}+2 \mathrm{H}_{\mathrm{M}}^{+} .
$$

Sum (reversal steps 1 and 2):

$$
\mathbf{O}+\mathbf{2} c^{3+}+2 \mathrm{H}_{2} \mathrm{O}+\text { energy }=\mathbf{P}+\mathbf{2} c^{2+}+4 \mathrm{H}_{\mathrm{M}}^{+} .
$$

In this case, the 'energy' term was represented by the high electrochemical proton gradient $\Delta \mu_{\mathrm{H}}^{+}$as well as the accompanying transmembrane potential $\Delta \psi$, and $\mathrm{H}_{M}^{+}$ represented the protons released from water hydrolysis. Experimental as well as computational assessments of this model have been characterized with solid alignment between the two conditions, although the experimental conditions demonstrated a lag in F state formation. This was attributed to a delay in the generation of the electrochemical proton gradient $\left(\Delta \mu_{\mathrm{H}}^{+}\right)$. To define the composition of these intermediate states, the $\mathrm{O}$ state is defined as an oxidized ferric-cupric state. Given 
the high electrochemical proton gradient and concentration of oxidized cytochrome $c$, the $\mathrm{O}$ intermediate can be driven to become the $\mathrm{F}$ intermediate, represented by a ferryl-cupric state with a dioxygen bound to the iron component, where an electron is transferred to cytochrome $c$ and coupled with proton translocation by COX [50]. The O-F transition has been confirmed using electron paramagnetic resonance (EPR) spectroscopy, where in addition to the formation of the $\mathrm{F}$ intermediate, $\mathrm{O}_{2}$ formation as well as a high reactivity of the intermediate with $\mathrm{CO}$ was observed $[54,55]$. Furthermore, the existence of the ferryl compound structure was proved using Raman spectroscopy in which the iron-oxygen vibration was observed at $790 \mathrm{~cm}^{-1}$ [56-58]. A second electron can also potentially be donated to cytochrome $c$ upon transition to the $\mathrm{P}$ intermediate, which recent information and spectroscopic analysis have suggested pairs the second electron with a ferryl heme/cupric structure that may also possess a neutral tyrosine radical [48-50]. In sum, this discussion has addressed several studies including optical spectroscopy, thermodynamic analysis, kinetic analysis, Raman vibrational spectroscopy, EPR spectroscopy, as well as computation/theoretical analysis that have collectively demonstrated both the existence of the $\mathrm{O}$, $\mathrm{F}$, and $\mathrm{P}$ intermediates, as well as the reduction of cytochrome $c$, in turn showing that proton-dependent heme group oxidation in COX sums the reversed reactions detailed in equations (7)(9), and can be reached using a high electrochemical proton gradient $\left(\Delta \mu_{\mathrm{H}}^{+}\right)$and electron acceptor potential $\left(E_{\mathrm{h}}\right)$ [48-50]. These intermediates and their associated processes that serve as the foundation of the partial reversal of COX activity then serve as the mechanisms for enabling the utilization of COX as the electron-generating element of the coupled protein system.

The detection of oxygen release from the COX protein was expected to be difficult to demonstrate because as COX reaches the ferryl/cupric/tyr radical condition, the path further backwards in the reaction pathway to the ferrous $-\mathrm{O}_{2}$ cuprous state involves no electron transfer out of the heme/copper site [50]. In fact, the remaining reactions only occur within it. Documented internal charge transfer reactions to equilibrate charge transfer imbalances, as well as full protein reversal (oxygen release), have been well characterized and studied [23-26]. Furthermore, internal electron transfer is not coupled to charge translocation across the membrane which was observed with the previous reactions, and therefore could not be driven backwards simply by the electrochemical proton gradient as observed with the two earlier reactions [48-50]. The driving force (high electrochemical proton gradient) and the coupling of charge translocation to electron movement (out of the molecule) are key factors why the first two steps of COX reversibility are possible and measurable via cytochrome $c$ reduction and electron harvesting.

While this study has demonstrated a light-dependent reversal of COX through observed current increases, it is unclear as to which intermediate state was reached in this case. We do believe, however, that either 1 or 2 electrons could be released by the reversal of the $\mathrm{a} 3 / \mathrm{Cu}_{\mathrm{B}}$ site of each COX molecule per partial reversal cycle depending on whether the protein underwent a transition to the $\mathrm{F}$ intermediate (one electron) or P intermediate (two electrons), with the remaining electrons undergoing an internal charge transfer not coupled to proton translocation $[48,50]$. Based upon the range of current increases obtained, and given that one ampere is equivalent to $6.24 \times 10^{18}$ charged particles moving through a surface in one second, it was believed that the current increase due to partial COX reversal was due to the activity of $10^{11}-10^{13}$ proteins. This value is substantially lower than the number of COX proteins added into the solution [21], indicating in a similar fashion to the BR monomers that a portion of the injected protein was not reconstituted. While it could be determined that our system was capable of producing proteinbased microampere outputs, continued efforts to optimize protein reconstitution to injection ratios may offer higher current outputs.

\subsection{Polymer membrane properties and transmembrane water transport}

The capacity for water transport through the polymer has been documented in the literature, although values observed in the polymer have been observed to be approximately one order of magnitude lower in the polymer compared with conventional lipid systems [59, 60]. With respect to this composite vesicle device, it was important to establish that water molecules could indeed pass through the membrane in order to replenish COX-induced oxidized water molecules which served as the electron source. In addition, BR-functionalized polymer membranes were previously observed to reduce the electro-osmotic drag across Nafion ${ }^{\circledR}$ membranes [6, unpublished data]. Furthermore, measurements were performed whereby external salt gradients were developed in order to elicit water transport through the polymer in order to equilibrate internal/external solute concentration conditions (figure 9). Addition of the gradient to vesicle solutions resulted in gradual decreases in absorbance over time in comparison to blank samples which produced a negligible change in absorbance. These responses may have served as indications of transmembrane water transport that was believed to have resulted in reduction in vesicle size or possible collapse to explain the absorbance changes, similar to experiments observed in [61, 62]. Correlations have been established between membrane thickness, the polymer molecular weight, and mechanical stability, whereby an increased membrane thickness and molecular weight, commonly found in block copolymers, increases stability and decreases fluidity [59, 63, 64]. Furthermore, polymer vesicle characterization (giant vesicles, $\sim 10 \mu \mathrm{m}$ ) studies using single vesicle micromanipulation techniques were also able to demonstrate that increasing molecular weight resulted in increased viscosity of the membranes, also associated with decreased fluidity [65-67]. These studies also revealed that the decreases in polymeric fluidity were most pronounced in cases where chain lengths were long enough to become tangled or intertwined. However, the tangling of these chains as a result of the larger thickness, $d$, then contributed to an increased rupture resistance. These properties, then, have also been correlated with the fact that the increased membrane stability results in decreased water transport through the membrane [59]. Conventional lipid systems were described as having properties ( $\mathrm{MW}=10^{2}-10^{3}$ daltons) consisting of increased fluidity and decreased stability given their lower molecular weights and decreased thicknesses [68]. This 
would explain the mechanism of why lipid membranes are damaged or ruptured more easily. The lower thickness value $d$ of lipid systems makes the system more susceptible to irreversible rupture from defects or systemic fluctuations. To address the challenges of combining the robustness of polymer systems with lipid-like fluidity, we fabricated a low molecular weight copolymer of $\sim 5000$ daltons in which the $d$ value was reduced to resemble those typically observed with lipids. To enhance the polymeric resistance to fluctuations from the environment and any induced defects, UV-reactive endgroups, as previously mentioned, were integrated with the 'A' blocks of the polymer to enhance mechanical stability as confirmed through irreversible rupture trials. The predesigned molecular weight coupled with the crosslinking capabilities enabled our membrane to possess the advantages of both the lipids and polymers due to its optimized fluidity and stability [40]. This would explain the mechanism for the observed rapid transmembrane water transport observed during the aforementioned vesicle/absorbance testing, as well as increased resistance to irreversible rupture $(\sim 1.5 \mathrm{~V}$ crosslinked) over lipid systems $(\sim 500 \mathrm{mV})$.

\section{Conclusions}

A synthetic biology-based approach towards the synthesis of protein-functionalized polymeric technology has been demonstrated in this work. The polymeric membranes themselves have served as a key platform engineering material for the technological development of these systems as evidenced by our custom engineering of the membrane thicknesses to mimic those found in natural biomembranes. These thicknesses $(\sim 4 \mathrm{~nm})$ were also believed to contribute to the ability for transmembrane water transport through the mimicry of lipid membrane fluidity. In addition, UVcrosslinkable endgroups were integrated to enhance their demonstrated superior robustness over conventional lipid systems that have been used for basic characterization of single protein functionality. Furthermore, their robustness and potential as bioengineering materials were confirmed through field-induced irreversible rupture trials as well as Langmuir film collapse tests. Analysed individually, the bacteriorhodopsin and cytochrome $c$ oxidase proteins function as proton pumps. However, by engineering these proteins as active components into block copolymer membranes, we have utilized BR as a light-dependent switch to alter the mechanistic functionality of COX to partially reverse its proton pumping activity and release electrons. As such, their functionalities were coordinated and coupled towards light-dependent current generation from composite vesicles. Following the demonstration of light-activated proton pumping by $\mathrm{BR}$, the gradient-dependent current production of both the composite vesicles and COX-only vesicles was also observed. This was seen through observations of on/off states for the composite protein-functionalized vesicles corresponding to light/dark environments. Current increases as high as $10 \mu \mathrm{A}$ were observed. As such, the harnessing of energy conversion processes from proteins has been explored as a potentially impacting process that may be exploited for practical use. Future studies will consider methodologies of vesicle fabrication to increase the yield of functional vesicles, as well as increasing electron harvesting through novel electrode configurations to enhance the conversion efficiency of the system. This study has demonstrated an important advance towards developing novel power sources and protein-based devices.

\section{Acknowledgments}

This work was supported by DARPA grant N66001-02-C8402. The authors gratefully acknowledge the FergusonMiller group for the engineered COX strains. The authors would also like the thank Mr Hyo-Jick Choi for TEM assistance.

\section{References}

[1] Stoeckenius W and Lozier R H 1974 J. Supramol. Struct. 2769

[2] Stoeckenius W 1979 Structure of biological membranes Biochem. Biophys. Acta $\mathbf{5 0 5} 215$

[3] Spudich J L and Bogolmolni R A 1983 J. Biophys. 43243

[4] Spudich J L and Bogolmolni R A 1984 Nature 312509

[5] Lee H, Ho D, Schmidt J and Montemagno C D 2003 IEEE Proc. Nanotechnol. 2733

[6] Ho D, Chu B, Lee H and Montemagno C D 2004 Nanotechnology 151084

[7] Spudich J L and Bogolmolni R A 1984 Annu. Rev. Biophys. Chem. 17193

[8] Hiser C, Mills D A, Schall M and Ferguson-Miller S 2001 Biochemistry 401606

[9] Allen J P, Feher G, Yeates T O, Rees D C, Deisenhoffer J, Michel H and Huber R 1986 Proc. Natl Acad. Sci. USA 83 8589

[10] Yasuda R, Noji H, Kinosita K Jr and Yoshida M 1998 Cell 931117

[11] Hong F T 1999 Prog. Surf. Sci. 621

[12] Racker E and Stoeckenius W 1974 J. Biol. Chem. 249662

[13] Nardin C, Winterhalter M and Meier W 2000 Langmuir 167708

[14] Nardin C, Widmer J, Winterhalter M and Meier W 2001 Eur. Phys. J. E 4403

[15] Meier W, Nardin C and Winterhalter M 1999 Angew. Chem. Int. Edn 394599

[16] Vsevolodov N 1998 Biomolecular Electronics-An Introduction via Photosensitive Proteins (Boston, MA: Birkhauser)

[17] Das R et al 2004 Nano Lett. 41079

[18] Kim H, Mano N, Zhang Y and Heller A 2003 J. Electrochem. Soc. 150 A209

[19] Lee H, Ho D, Kuo K and Montemagno C D 2004 Proc. Electrochem. Soc. AH1 631

[20] Celedon A 2005 Masters Thesis Department of Bioengineering, University of California at Los Angeles

[21] Ho D, Chu B, Lee H, Kuo K and Montemagno C D 2004 Proc. Electrochem. Soc. AH1 621

[22] Wikstrom M 1981 Proc. Natl Acad. Sci. USA 784051

[23] Barker P D and Ferguson S J 1999 Struct. Fold. Des. 7 R281

[24] Saraste M 1999 Science 2831488

[25] Schultz B E and Chan S I 2001 Annu. Rev. Biophys. Biomol. Struct. 3023

[26] Bloch D, Belevich I, Jasaitis A, Ribacka C, Puustinen A, Verkhovsky M I and Wikstrom M 2004 Proc. Natl Acad. Sci. USA 101529

[27] Graff A, Sauer M, Van Gelder P and Meier W 2002 Proc. Natl Acad. Sci. USA 995064

[28] Hazard A and Montemagno C D 2002 Arch. Biochem. Biophys. 407 117-24

[29] Ho D, Chu B, Schmidt J J, Brooks E K and Montemagno C D 2004 IEEE Trans. Nanotechnol. 3256 
[30] Scheffler I 1999 Mitochondrial electron transport and oxidative phosphorylation Mitochondria (New York: Wiley) chapter 5

[31] Holst G C (ed) 1998 CCD Arrays Cameras and Displays 2nd edn (Bellingham, WA: SPIE Press)

[32] Pata V and Dan N 2003 Biophys. J. 852111

[33] De Sanctis G, Maranesi A, Ferri T, Poscia A, Ascoli F and Santucci R 1996 J. Protein Chem. 15599

[34] Bonanni B, Alliata D, Andolfi L, Bizzarri A and Cannistraro S 2004 Surface Science Research Development ed C Norris (New York: Nova Science Publishers, Inc.) p 1

[35] Bonanni B, Alliata D, Bizzarri A and Cannistraro S 2003 Chem. Phys. Chem. 41183

[36] Xi J, Ho D, Chu B and Montemagno C D 2005 Adv. Funct. Mater. 151233

[37] Lea C 1999 Thesis Hampton-Syndney College online at http://cator.hsc.edu/ leac/352page.html\#experimnt

[38] Wang J 2000 Analytical Electrochemistry (New York: Wiley) chapter 2

[39] Happe M et al 1977 Biochim. Biophys. Acta 465415

[40] Kimura Y, Vassylyev D G, Miyazawa A, Kidera A Matsushima M, Mitsuoka K, Murata K, Hirai T and Fujiyoshi Y 1997 Nature $\mathbf{3 8 9} 206$

[41] Luecke H B, Richter H-T and Lanyi J K 1998 Science 2801934

[42] Iwata S, Ostermeiner C, Ludwig B and Michel H 1995 Nature 376660

[43] Oesterhelt D 1998 Curr. Opin. Struct. Biol. 8 489-500

[44] Chapman J B, Johnson E A and Kootsey J M 1983 J. Membr. Biol. 74139

[45] Hammes G G 1982 Proc. Natl Acad. Sci. USA 796881

[46] Wikstrom M, Krab K and Saraste M 1981 Annu. Rev. Biochem. 50623

[47] Muneyaki E and Fukami T A 2000 Biophys. J. 781166

[48] Wikstrom M and Morgan J E 1992 J. Biol. Chem. 26710266
[49] Wikström M and Saari H T 1977 Biochim. Biophys. Acta 462 347

[50] Wikstrom M 2004 personal communication

[51] Wikstrom M 1988 Chem. Scr. 28A 71

[52] Devault D 1971 Biochim. Biophys. Acta 225193

[53] Chance B, Erecinska M, Dutton P L, Wilson D F and DeVault D 1971 Energy Transduction in Respiration and Photosynthesis ed E Quagliariello, S Papa and C S Rossi (Italy: Bari) p 3

[54] Blair D F, Witt S N and Chan S I 1985 J. Am. Chem. Soc. 1077389

[55] Orii Y 1988 Ann. New York Acad. Sci. 550105

[56] Varotsis C and Babcock G T 1990 Biochemistry 297359

[57] Ogura T, Takahashi S, Shinzawa-Itoh K, Yoshikawa S and Kitagawa T 1990 J. Biol. Chem. 26514721

[58] Han S, Ching Y C and Rousseau D L 1990 Nature 34889

[59] Discher D and Eisenberg A 2002 Science 297967

[60] Nardin C and Meier W 2002 Rev. Mol. Biotech. 9017

[61] Jacob-Dubuisson F, El-Hameli C, Saint N, Guedin S, Willery E, Mollei G and Locht C 1999 J. Biol. Chem. 27437731

[62] Discher B M 1999 Science 2841143

[63] Aranda-Espinoza H, Bermudez H, Bates F S and Discher D E 2001 Phys. Rev. Lett. 87208301

[64] Bermudez H, Brannan A K, Bates F S, Hammer D A and Discher D E 2002 Macromolecules 35 8203-8

[65] Evans E A and Skalak R 1980 Mechanics and Thermodynamics of Biomembranes (Boca Raton, FL: CRC Press)

[66] Lee J C-M, Santore M, Bates F S and Discher D E 2002 Macromolecules 35323

[67] Bloom M, Evans E and Mouritsen O G 1991 Q. Rev. Biophys. 24293

[68] Bangham A D 1993 Chem. Phys. Lipids 64275 